\title{
Evaluación del Video Head Impulse Test (V-Hit) en el diagnóstico del neurinoma del acústico
}

\author{
Evaluation of video Head Impulse Test (v-HIT) \\ in the diagnosis of acoustic neuroma
}

Mauricio Silva $C^{1}$, Roberto Arias $A^{1}$, Cristián Carriel P², Homero Sariego $\mathbf{R}^{3}$.

\begin{abstract}
RESUMEN
Introducción: La gran mayoría de lesiones que comprometen el conducto auditivo interno corresponden a schwannomas vestibulares (90\%). Dentro de las nuevas pruebas diagnósticas del sistema vestibular aparece la prueba de impulso cefálico y su registro con el video Head Impulse Test (v-HIT) que permite el estudio de todos los canales semicirculares.

Objetivo: Comparar el v-HIT para canales semicirculares laterales con las pruebas calóricas clásicas registradas mediante videonistagmografía en el comportamiento de los neurinomas intracanaliculares y evaluar su utilidad como test diagnóstico.

Material y método: Dieciocho pacientes fueron reclutados con diagnóstico de neurinomas, confirmado mediante resonancia magnética y estudios con pruebas calóricas clásicas y v-HIT para canal horizontal.

Resultados: Los resultados anormales de asimetría de ganancia y de sacadas correctivas fue de 13 pacientes (72\%) y con hipoexcitabilidad en la prueba calórica fue de 9 pacientes (50\%), con una sensibilidad de la prueba de $72 \%$ para vHIT y de $50 \%$ para la prueba calórica al compararlo con la resonancia magnética.

Conclusión: El v-HIT es una herramienta complementaria a las pruebas calóricas y debe ser incluida como test diagnóstico dentro de la batería de exámenes para evaluar el sistema vestibular en forma completa mediante un vestibulograma.
\end{abstract}

Palabras clave: Schwannomas, v-HIT, vestibulograma.

\begin{abstract}
Introduction: The majority of injuries involving the internal auditory canal correspond to vestibular schwannomas (90\%). Within the new diagnostic tests for the vestibular system appears the Head Impulse test and the video head impulse test (v-HIT) which allows the studying of all the semicircular canals.
\end{abstract}

\footnotetext{
Médico Servicio de Otorrinolaringología. Hospital del Salvador.

Tecnólogo Médico-ORL. Servicio Otorrinolaringología. Hospital del Salvador.

3 Médico Servicio de Otoneurología. Instituto Neurocirugía Dr. Asenjo.
}

Recibido el 15 de junio de 2015. Aceptado el 9 de agosto de 2015. 
Aim: Compare the v-HIT lateral semicircular canals with classical caloric tests recorded by videonistagmography (VNG) in the behavior of intracanalicular neuroma and evaluate its usefulness as a diagnostic test.

Material and method: Eigtheen patients were enrolled with a diagnosis of schwannomas, confirmed by MRI and studied classical caloric tests and v-HIT for horizontal channel.

Results: Abnormal results of asymmetry gain and corrective saccades was 13 patients (72\%) and hypoexcitability in the caloric test was 9 patients (50\%), with a test sensitivity of $72 \%$ for vHIT and $50 \%$ for caloric test compared with MRI.

Conclusions: The v-HIT is a complementary tool to caloric tests and should be included as a diagnostic test within the battery of tests to evaluate the vestibular system in complete form by vestibulogram.

Key words: Schwannomas, v-HIT, vestibulogram.

\section{INTRODUCCIÓN}

Se han descrito diversas lesiones que comprometen el conducto auditivo interno (CAl), la gran mayoría de los casos corresponde a schwannomas vestibulares $(90 \%)^{1,2}$, con una incidencia estimada de 1 en $100.000^{1}$ de manera más frecuente entre la cuarta y sexta década de la vida y sin predominio de sexo. Se trata de una neoplasia de origen neural, benigna, originada en las células de Schwann de los nervios vestibulares superior e inferior, siendo raro su origen en el nervio facial y menor aún en el coclear².

La sospecha clínica de neurinoma se inicia ante la presencia de hipoacusia sensorioneural asimétrica, lentamente progresiva ${ }^{3} 0$ súbita, en el menor de los casos (1\%-2\%) algún grado de desequilibrio o inestabilidad y vértigo ${ }^{4}$. Un paciente con esta sintomatología deber ser estudiado funcionalmente con examen audiométrico básico completo, electronistagmografía de las pruebas vestibulares y complementariamente impedanciometría con estudio del reflejo acústico. Una curva de hipoacusia sensorioneural descendente con pruebas supraliminares de neuropatía y/o hipo 0 arreflexia vestibular unilateral, mayor aún sintomática, orientan la atención diagnóstica ${ }^{5}$. Si este estudio que tiene baja correlación diagnóstica en cuanto a especificidad y sensibilidad ${ }^{6}$, tiene algunos indicadores de lesión neural, debe solicitarse una resonancia magnética de encéfalo con énfasis en fosa posterior y secuencia T1 3D Wats y T2 cóclea, que definitivamente hace el diagnóstico de la lesión ${ }^{2,9}$ que según los distintos protocolos y estudios muestran una sensibilidad y especificidad sobre el $95 \%{ }^{10}$.

Frente a un diagnóstico radiológico de masa en el conducto auditivo interno (CAI), debemos abocarnos en el diagnóstico neural funcional. Si la audiometría está dentro de límites normales se debe estudiar la audición subclínica con emisiones otoacústicas producto de distorsión (EOA-PD) y eventualmente potenciales evocados auditivos de tronco cerebral (PEATC), sabiendo que las pruebas calóricas son indicadores del daño del nervio vestibular superior a nivel de estímulos umbrales, podemos complementarlos con estimulaciones de alta frecuencia como el test de impulso cefálico con registro ( $\mathrm{v}$-HIT, video head impulse test), este examen también tiene importancia en el diagnóstico del nervio singular. El nervio vestibular inferior es examinado por un reflejo vestibuloespinal cuya aferencia es auditiva y la respuesta una relajación del músculo esternocleidomastoideo ipsilateral registrado con electrodos de superficie cVEMP (cervical vestibular evoked myogenic potentia).

Dentro de las nuevas pruebas diagnósticas del sistema vestibular aparece el potencial evocado miogénico vestibular cervical (cVEMP) para estudio del nervio vestibular inferior y la prueba de impulso cefálico (HIT) que permite el estudio de todos los canales semicirculares $y$, por ende, de ambos nervios vestibulares. La prueba de impulso cefálico fue descrita en 1988 por Halmagyi ${ }^{10}$, ésta evalúa la eficiencia del reflejo vestíbulo-oculomotor $(\mathrm{RVO})^{12}$. Una manera objetiva de evaluar el RV0 es a través del video head impulse test ( $\mathrm{V}-\mathrm{HIT}$ ). El video head impulse test $(\mathrm{vHIT})^{13}$ se basa en el 
registro de los movimientos oculares con el uso de cámaras de alta velocidad que logran captar los movimientos compensatorios durante el impulso cefálico y permite evaluar por separado ambos canales semicirculares, a diferencia de la silla rotatoria12. Una asimetría en el RVO traduce una alteración a nivel de canales semicirculares (función angular) y por lo tanto una hipofunción vestibular. La presencia de sacadas oculares correctivas durante 0 después del movimiento cefálico, encubiertas y descubiertas respectivamente, también traduce una alteración del RVO ${ }^{12,16}$. Es así como ganancia disminuida y presencia de sacadas correctivas traducen una alteración del RVO.

El v-HIT es una prueba sencilla, rápida de realizar, objetiva y que no genera síntomas molestos en los pacientes y que además permite evaluar la función de los 6 canales semicirculares por separado ${ }^{13,14}$. De ahí entonces su utilidad en la detección de patologías que afectan al nervio vestibular, tanto del superior como el inferior, como son los neurinomas ${ }^{15}$.

El diagnóstico precoz de neurinoma resulta fundamental para pesquisar pequeñas lesiones, lo que se correlaciona con menor morbimortalidad derivada de su tratamiento. El menor tamaño de la lesión al momento del diagnóstico se traduce en mejores resultados tanto de la audición como la función del nervio facial ${ }^{6}$.

\section{OBJETIVO}

El objetivo del presente trabajo es comparar el V-HIT para canales semicirculares laterales con las pruebas calóricas clásicas registradas mediante videonistagmografía (VNG) en el comportamiento de los neurinomas intracanaliculares y evaluar su utilidad como test diagnóstico. Correlacionar los hallazgos de v-HIT versus pruebas calóricas en la ubicación y tamaño de los neurinomas.

Conocer las características clínicas y audiológicas de pacientes portadores de neurinomas para dar la orientación necesaria con respecto a la acción médica más adecuada.

\section{MATERIAL Y MÉTODO}

Es un estudio prospectivo de tipo descriptivo en el que se incluyen pacientes con diagnóstico de neurinoma unilateral no tratados y con diagnóstico confirmado mediante resonancia magnética con secuencia T2 y T1 con contraste con gadolinio de cóclea y fosa posterior.

Se escogió una muestra por criterio de factibilidad que consistía en pacientes con diagnóstico de neurinoma del acústico, diagnosticados durante la fecha de realización del estudio. Un total de 18 pacientes fueron reclutados con diagnóstico de neurinomas atendidos en el Hospital Salvador y del Instituto de Neurocirugía (INCA) durante el periodo diciembre 2012 y septiembre de 2014. A estos pacientes se les realizó audiometría de tonos puros con discriminación de la palabra y pruebas supraliminares (LDL), pruebas calóricas con videonistagmografía (VNG) y pruebas de V-HIT. La resonancia magnética fue realizada e informada en el INCA, la que determinó tamaño y ubicación de la lesión tumoral. Los neurinomas fueron medidos en milímetros según el eje de mayor diámetro y clasificados de acuerdo a la clasificación KOOS.

Se realizó en todos los pacientes prueba de impulso cefálico asistida por video (v-HIT) para el canal horizontal, bajo consentimiento informado. Para esta medición se utilizó un equipo marca Interacoustics, modelo EyeSeeCam ${ }^{\mathrm{TM}}$ año 2013 con derivación para canales laterales.

Para realizar el examen se colocaron las gafas del equipo, ajustadas a cada paciente, sentado aproximadamente a un metro de distancia de una pared, la cual tenía una marca. Manteniendo una inclinación en aproximadamente de $30^{\circ}$ sobre la horizontal se realizan al menos 20 movimientos cefálicos en plano horizontal (yaw). Los impulsos cefálicos se realizaron horizontalmente a derecha e izquierda con ángulo de rotación de $10^{\circ}$ a $15^{\circ}$ (por lo menos $10^{\circ}$ ) en formas aleatorias, rápidas, cortas en duración e inesperadas evitando que fuesen predecibles para el paciente. Estos impulsos se aplicaron manualmente por un mismo examinador experimentado. Las velocidades de los ojos y la cabeza $[\%$ s] fueron capturados a las 40, 60 y 80 ms después del inicio del impulso cefálico y se tomó un promedio de ellos. La velocidad de la ganancia del RVO está calculado por la relación entre el promedio de la velocidad del ojo $[\%$ s] y el promedio de la velocidad de la cabeza $[\%$ s]. Se consideraron sacadas correctivas encubiertas (covert saccades) aquellas que ocurrieron antes que termine el movimiento cefálico y descubiertas (overt sacca- 
des) aquellas que ocurrieron en forma posterior. Se registraron tanto formas de las curvas como valores cuantitativos de la ganancia del RVO, su asimetría en la ganancia del RVO entre ambos lados, las sacadas correctivas, encubiertas y descubiertas (covert-overt saccades). Se consideró un v-HIT patológico frente a ganancias menores a 0,77 , asimetrías de repuestas (mayor a 7\%), y/o presencia de covert-overt saccades acorde a la literatura internacional ${ }^{13,14}$.

Las pruebas calóricas se realizaron en una posición supina con elevación de la cabeza a 30 grados con agua con temperaturas de $30^{\circ}$ y $44^{\circ}$, en ciertos pacientes se ocupó aire. Los movimientos oculares horizontales se registraron con un sistema binocular de video oculografía (VO 425TM, Interacoustics, Dinamarca).

En la VNG se evalúo la asimetría y la dirección preponderante en función de la velocidad de componente lento del nistagmo medido en grados por segundos considerando como patológico una diferencia mayor al $25 \%$.

Se excluyeron a pacientes con patología central, patología cervical, uso de fármacos de acción central que enlentezcan el RVO, presencia de estrabismo o pérdida severa de la agudeza visual y pacientes con crisis recientes vestibulares de menos de 2 semanas de evolución.

El análisis estadístico se realizó con software estadístico SPSS para Windows 14.0.0.

Este estudio fue aprobado por el Comité de Ética local de nuestro hospital.

\section{RESULTADOS}

Un total de 18 pacientes fueron evaluados, el test de Shapiro-Wilk indicó que los valores tenían una distribución normal. De éstos $66 \%$ corresponden a mujeres. El promedio de edad fue de 55,7 años ( \pm desviación estándar 11,61 años, con IC 95\%: [49,95 - 61,5]. En su mayoría correspondieron a neurinomas dependientes del nervio vestibular superior $(66 \%)$. Con respecto a los resultados audiométricos el promedio de pérdida auditiva en el oído afectado fue de 50,8 dB. El tipo de curva audiométrica más frecuente fue la descendente (72\%). La discriminación promedio fue de $72 \%$ y $50 \%$ de los pacientes al momento del diagnóstico presentaba audición no útil.

Los resultados anormales de asimetría de ganancia y de sacadas correctivas fue de 13 pacientes (72\%) y con hipoexcitabilidad en la prueba calórica fue de 9 pacientes (50\%). Esto corresponde a una sensibilidad de la prueba de $72 \%$ para vHIT y de $50 \%$ para la prueba calórica al compararlo con la resonancia magnética (Tabla 1). El lado con valores de prueba calórica y vHIT anormales siempre fue en el lado afectado con el schwanoma vestibular, basado en la RM.

Al comparar las pruebas calóricas versus el V-HIT, no se encontró correlación entre la valores alterados de la prueba calórica (menor 25\%) y valores alterados de la ganancia (mayor al $7 \%$ ), $(\mathrm{R}=0,19 \mathrm{p}>0,05)$ (Figura 1). Así como tampoco correlación entre prueba calórica alterada y v-HIT alterado, asimetría y sacadas correctivas $(R=0,349$ у $p=0,156$ ) (Tabla 2). Un v-HIT anormal se encontró en 13 casos (72\%) y de ellos 7 casos tenían pruebas calóricas alteradas. De los 9 pacientes con pruebas calóricas alteradas 2 de ellos no tenían alteración del v-HIT, lo que equivale a una sensibilidad de $77,7 \%$ del v-HIT al tener como referencia las PC (Tabla 3). Del total de pacientes evaluados 13 tenían sacadas correctivas y de ellos 5 tenían ganancia alterada, no

Tabla 1

\begin{tabular}{|lccc|}
\hline PRUEBA CALORICA & V-HIT & & \\
\hline$\%$ Hipoexc & Normal & Anormal & Total \\
\hline$<25$ & 3 & 6 & 9 \\
& $(16,6 \%)$ & $(33,3 \%)$ & $(50 \%)$ \\
$>25$ & 2 & 7 & 9 \\
& $(11,1 \%)$ & $(38,9 \%)$ & $(50 \%)$ \\
TOTAL & 5 & 13 & 18 \\
& & $(72,2 \%)$ & \\
\hline
\end{tabular}




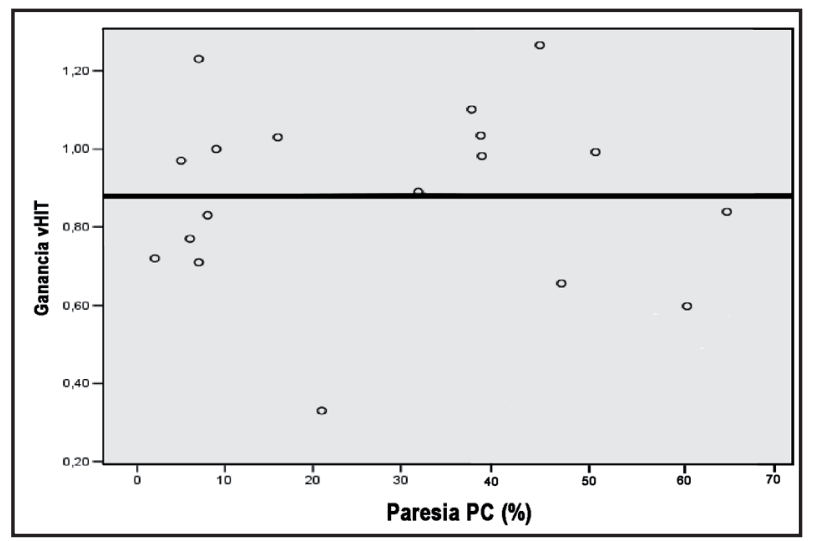

Figura 1.

Tabla 2.

\begin{tabular}{|lll|}
\hline Correlación & \multicolumn{1}{c|}{$\mathrm{R}$} & $\mathrm{p}$ \\
\hline PC y v-HIT alterado & 0,349 & 0,156 \\
PC y ganancia & 0,19 & 0,940 \\
V-HIT y tamaño tumoral $(\mathrm{mm})$ & 0,334 & 0,176 \\
\hline
\end{tabular}

encontrando ningún paciente con ganancia alterada y sin sacadas correctivas. Del total de pacientes evaluados, 15 de ellos tienen alteración en la PC y/o en el v-HIT. De los 18 pacientes, sólo 3 resultaron con prueba calórica normal y v-HIT normal, al realizarles el VEMPc sólo uno resultó asimétrico.

No se encontró correlación entre el tamaño del tumor en milímetros y la alteración del v-HIT ( $R$ $=0,334$ y $p=0,176$ ) (Tabla 2).

De nuestro grupo estudiado 4 pacientes habían sido diagnosticados como dependientes del nervio vestibular inferior, dado que la prueba calórica era simétrica. De ellos sólo uno tenía el v-HIT normal, tanto en ganancia como en sacada correctiva y presentaba VEMPc asimétrico.

\section{DISCUSIÓN}

Ambas pruebas, la calórica como el v-HIT son exámenes para evaluar principalmente la función vestibular periférica. Éstas se basan en detectar una alteración del RVO. Diversas patologías periféricas como la neuritis vestibular, el hidrops endolinfático, intoxicación por gentamicina u otros ototóxicos
Tabla 3.

\begin{tabular}{|lrc|}
\hline & S & E \\
\hline V-HIT con RM & $72,2 \%$ & $100 \%$ \\
PC con RM & $50 \%$ & $100 \%$ \\
\hline
\end{tabular}

alteran el RV0 ${ }^{16}$. Lesiones retrococleares como el schwanoma vestibular que alteran el RVO pueden ser detectables con el v-HIT o con las pruebas calóricas, debido a compresión de neuronas que codifican diferentes frecuencias de estimulación, por crecimiento tumoral o por disminución del flujo sanguíne ${ }^{17}$.

En nuestro estudio la gran mayoría de nuestros pacientes mostró una alteración de los exámenes en lado afectado por el schwanoma, sólo 3 casos presentaron exámenes normales en el lado involucrado. El v-HIT mostró una mayor sensibilidad (72\%) que la prueba calórica (50\%) en la detección de neurinomas al compararlos con la RM, similar a lo publicado en otras series ${ }^{14,16}$.

Ambas pruebas evalúan el sistema vestibular pero lo hacen a frecuencias distintas. Mientras la prueba calórica evalúa primariamente el RVO a través del canal semicircular lateral a baja frecuencia, en un rango aproximado de $0,025 \mathrm{~Hz}$, el v-HIT muestra sus deficiencias a alta frecuencia, en un rango de 2 a $5 \mathrm{~Hz}$. En la vida cotidiana los tres canales son estimulados en forma simultánea en un rango de frecuencia que es más cercano a lo censado por el v-HIT. Ambas pruebas resultan 
entonces complementarias para la evaluación del sistema vestibular, así por ejemplo en un neurinoma una prueba calórica puede estar abolida pero puede tener un RVO conservado a altas frecuencias con un v-HIT normal, y viceversa. En los neurinomas todas las frecuencias de estimulación pueden alterarse. En nuestro estudio el rango de frecuencias altas del RVO de los neurinomas fueron las más afectadas. Para identificar el rango de frecuencia baja y alta del RVO, son necesarias ambas pruebas, la calórica y VHIT. Aparentemente los rangos de las distintas frecuencias del RVO son censados por distintos subgrupos de células ciliares, los grupos de células ciliares tipo I codificarían para estímulos de alta frecuencia y las tipos II para bajas frecuencias ${ }^{18}$. Nuestra asociación entre prueba calórica asimétrica, $>25 \%$ y un v-HIT alterado, fue de $77,7 \%$, mayor a lo que otros autores han reportado en neuronitis vestibular $(33 \%)^{19}$.

En nuestro estudio el tamaño del tumor no se correlacionó con mayor alteración del v-HIT así como tampoco con mayor asimetría de la prueba calórica como ha sido descrito en otros trabajos ${ }^{20}$. Esto puede ser explicado por el lento crecimiento del schwanoma con deterioro crónico del sistema vestibular lo que produce una compensación vestibular a nivel central generando niveles de ganancias bajas (menores a 1) en ambos lados.

Las sacadas correctivas (encubiertas y descubiertas) están presentes en un mayor número de portadores de neurinomas $(66,6 \%)$ al compararla con los pacientes que tienen sólo asimetría de la ganancia $(27,7 \%)$, por lo que se podría plantear como un patrón más sensible en la detección de patología vestibular. Algunos autores han demostrado una pobre relación entre asimetría de la ganancia y paresia vestibular ${ }^{22}$. Hay que considerar también que las ganancias de ambos lados, ipsi y contralesional, pueden estar disminuidas y por ende tener menor asimetría, dependiendo en qué momento de la evolución de su vestibulopatía fue evaluado.

En general, Ios resultados de nuestro estudio y los datos publicados en la literatura muestran que ambas pruebas son necesarias para evaluar el espectro completo de frecuencia del RVO, en caso de deterioro. La importancia clínica radica en que a ciertas frecuencias el RVO se puede encontrar normal y en otras alteradas. Algunos autores han propuesto que la recuperación de frecuencias altas ocurre antes que las bajas en las neuronitis vestibulares, en pacientes evaluados con v-HIT y PC ${ }^{21}$. Surge entonces la necesidad de evaluar en forma completa el RVO analizando todas sus frecuencias y con ello conocer su deterioro, a través de un vestibulograma. El concepto de vestibulograma, recientemente introducido, permite evaluar el RVO en todas sus frecuencias de estimulación y con ello conocer el estado del sistema vestibular en forma más completa. También nos permitiría conocer el estado de evolución de las distintas patologías vestibulares.

\section{CONCLUSIÓN}

La prueba de impulso cefálico registrada con video (v-HIT) es un nuevo test diagnóstico objetivo, sencillo, rápido de realizar, y que no genera síntomas molestos para los pacientes a diferencia de las pruebas calóricas. Permite además evaluar los canales superior y posterior además del lateral, lo que nos da más información del sistema vestibular.

Para la detección del deterioro del RVO son necesarias tanto la prueba calórica como el v-HIT en neurinomas. El v-HIT no reemplaza a la prueba calórica sino que es complementaria y no excluyente. Por lo tanto, al evaluar el sistema vestibular en forma inicial debería estudiarse con las pruebas calóricas clásicas y con v-HIT, incluyendo el estudio de todos los canales 0 , en su defecto, complementarlo con un VEMPC.

Nuestros hallazgos muestran que el v-HIT se altera con mayor frecuencia que las $P C$ en schwanomas vestibulares lo que sugiere que se alteran mayoritariamente las frecuencias altas del RVO, lo que puede tener cierta importancia en el tratamiento. El tamaño del tumor, en nuestro estudio no tiene relación con la alteración del v-HIT ni con las pruebas calóricas.

El v-HIT es una herramienta útil y complementaria a las pruebas calóricas y debe ser incluida como test diagnóstico dentro de la batería de exámenes para evaluar el sistema vestibular. Además permite explorar la progresión de los trastornos vestibulares en el tiempo.

Se recomienda realizar un análisis completo y dinámico del sistema vestibular mediante un vestibulograma que incluya PC y v-HIT en neurinomas, para realizar el análisis del rango completo de 
frecuencias del RVO en vestibulopatías periféricas agudas 0 crónicas.

Agradecimientos a la Dra. Mariana Bogado por su aporte en el análisis estadístico.

\section{BIBLIOGRAFÍA}

1. Dazert S, Aletsee C, Brors D, Miynski R, Sudhoff H, HiLdmann H, HeLms J. Rare tumors of the infernal auditory canal. Eur Arch Otorhinolaryngol 2005; 262: $550-4$

2. Stott C, Albertz A, Aedo C. Neurinoma del acústico (schwanoma vestibular): Revisión y actualización de la literatura. Rev Otorrinolaringol Cir Cabeza Cuello 2008; 68: 301-8.

3. Selesnick SH, Jackler RK, PItTs LW. The changing clinical presentation of acoustic tumors in the MRI era. Laryngoscope 1993; 103: 431-6.

4. Larach, A Lanas, C Napolitano, R Iñiguez, H Riveros. Experiencia en sospecha y diagnóstico del neurinoma del acústico. Rev Otorrinolaringol Cir Cabeza Cuello 2003; 63: 167-72.

5. Selesnick SH, Jackler RK. Clinical manifestations and audiologic diagnosis of acoustic neuromas. Otolaryngol Clin North Am 1992; 25(3): 521-51.

6. Morales C. Sospecha clínica de neurinoma del acústico y correlato imagenológico. Rev Otorrinolaringol Cir Cabeza Cuello 2002; 62: 6-12.

7. Morales C. Diagnóstico precoz del neurinoma del acústico. Rev Otorrinolaringol Cir Cabeza Cuello 1999; 59: 21-5.

9. Stucken EZ, Brown K, Selesnick SH. Clinical and diagnostic evaluation of acoustic neuromas. Otolaryngol Clin North Am 2012; 45(2): 269-84.

10. Halmagy G, CuRthoys I. A Clinical Sing of Canal Paresis. Arch Neurol 1988; 45: 737-9.

11. Wong BY, Capper R. Incidence of vestibular schwannoma and incidental tomography scans of patients from a direct referral audiology clinic. $J$ Laryngol Otol 2012; 126(7): 658-62.

12. Carriel C, Rojas M. Prueba de impulso cefálico: Bases fisiológicas y métodos de registro del reflejo vestíbulo oculomotor. Rev Otorrinolaringol Cir Cabeza Cuello 2013; 73: 206-12.

13. Weber K, Aw S, Todd M, Mc Garvie L, Curthoys I, HaLmagy G. Head Impulse Test in Unilateral Vestibular Loss: Vestibulo-ocular Reflex and Catchup Saccades. Neurology 2008; 70: 454-63.

14. Breinbauer H. Anabalón J. Experiencia en el uso video-impulso cefálico (vHIT) en la evaluación del reflejo vestíbulo-ocular para el canal semicircular horizontal. Rev Otorrinolaringol Cir Cabeza Cuello 2013; 73: 115-24.

15. Bıödow A, BLödow J, Bloching MB, Helbig R, WaLtheR LE. Horizontal VOR function shows frequency dynamics in vestibular schwannoma. Eur Arch Otorhinolaryngol 2014 May 1.

16. Blödow A, Pannasch S, Walther LE. Detection of isolated covert saccades with the video head impulse test in peripheral vestibular disorders. Auris Nasus Larynx 2013; 40: 348-51.

17. Hirvonen M, Aalto H, Petteri Hirvonen T. Motorized head impulse rotator in patients with vestibular schwannoma. Acta Otolaryngol 2008; 128 : 1215-20.

18. Straka H, Lambert FM, Pfanzelt S, Beraneck $M$. Vestibuloocular signal transformation in frequency-tuned channels. Ann N Y Acad Sci 2009; 1164: 37-44.

19. Mahringer A, Rambold HA. Caloric test and video-headimpulse: a study of vertigo/dizziness patients in a community hospital. Eur Arch Otorhinolaryngol 2013; 15.

20. Tringali S, Charpiot A, Ould MB, Dubreuil C, Ferber-Viart C. Characteristics of 629 vestibular schwannomas according to preoperative caloric responses. Otol Neurotol 2010; 31: 467-72.

21. Bartolomeo M, Biboulet R, Pierre G, Mondain M, Uziel A, Venall F. Value of the video head impulse test in assessing vestibular deficits following vestibular neuritis. Eur Arch Otorhinolaryngol 2013; 29.

22. Bell SL, Barker F, Heselton H, MacKenzie E, Dewhurst D, Sanderson A. A study of the relationship between the video head impulse test and air calorics. Eur Arch Otorhinolaryngol 2014; Nov 23.

Dirección: Mauricio Silva C.

Servicio de Otorrinolaringología, Hospital del Salvador.

Avenida Salvador 364. Providencia. Santiago.

E mail: msilvacar@yahoo.es 